\title{
PLZF inhibits proliferation and metastasis of gallbladder cancer by regulating IFIT2
}

\author{
Hui Shen ${ }^{1}$, Ming Zhan', Yonglong Zhang ${ }^{1}$, Shuai Huang ${ }^{1}$, Sunwang Xu', Xince Huang ${ }^{1}$, Min He ${ }^{1}$, Yanhua Yao ${ }^{2}$, \\ Mohan Man ${ }^{2}$ and Jian Wang ${ }^{1}$
}

\begin{abstract}
Gallbladder cancer (GBC) is a malignant cancer with very poor prognosis. Although promyelocytic leukemia zinc-finger protein (PLZF) was reported to be deregulated in numerous cancers and also relevant to clinical prognosis, its role in GBC progression has been little known. In this study, we found PLZF expression was decreased in GBC, correlating to advanced TNM stage, distant metastasis, and shorter overall survival. Moreover, ectopic PLZF expression in GBC cells (NOZ and GBC-SD) significantly reduced the cell proliferation, migration, and invasion. Consistently, overexpression of PLZF in xenograft mice model could suppress tumor growth and liver metastasis. Mechanical investigations verified PLZF could regulate the expression of cell cycle arrest-associated gene p21 and epithelial-mesenchymal transition (EMT)-related genes (E-cadherin and N-cadherin) in GBC cell lines. Importantly, PLZF remarkably increased the mRNA transcription of interferon-induced protein with tetratricopeptide repeat 2 (IFIT2) by increasing STAT1 protein level, a known factor involved in tumor progression. Furthermore, ablation of IFIT2 in PLZF overexpression cells abrogated the tumor-suppressive function of PLZF, at least partially, leading to impaired tumor growth and EMT program. These studies indicated PLZF inhibited the proliferation and metastasis via regulation of IFIT2. In conclusion, our study demonstrated PLZF could be a promising tumor biomarker for GBC, and also be a potential therapeutic target.
\end{abstract}

\section{Introduction}

Gallbladder cancer $(\mathrm{GBC})$ is a highly lethal and the most common biliary tract cancer, ranking the sixth leading cause of cancer-related death of digestive system ${ }^{1,2}$. Due to easier local infiltration and distant metastasis, GBC has an extremely poor prognosis with the median survival of 9.2 months and the 5 -year survival rate of $5 \%^{3,4}$. Given the lack of specific tumor marker and effective therapeutic targets for GBC, novel insight into the GBC progression contributes to the identification of potential targets for early diagnosis and therapy.

\footnotetext{
Correspondence: Jian Wang (dr_wangjian@126.com)

${ }^{1}$ Department of Biliary-Pancreatic Surgery, Renji Hospital, School of Medicine, Shanghai Jiao Tong University, 1630 Dongfang Road, Shanghai 200127, China ${ }^{2}$ Department of Biochemistry and Molecular Cell Biology, Shanghai Key Laboratory of Tumor Microenvironment and Inflammation, Institutes of Medical Sciences, School of Medicine, Shanghai Jiao Tong University, Shanghai 200025, China

Hui Shen and Ming Zhan contributed equally to this work.

Edited by M. Agostini.
}

One protein of Kruppel-like zinc-finger proteins family, promyelocytic leukemia zinc-finger protein (PLZF), also known as ZBTB16, was first discovered in acute promyelocytic leukemia as a fusion protein with the retinoic acid receptor $\alpha^{5,6}$. PLZF is involved in diverse cellular processes, particularly in stem cells self-renewal or differentiation, and immune cells development ${ }^{7}$. Nevertheless, the role of PLZF appeared to be controversial in tumor progression. Several studies showed PLZF was able to reduce cell growth and survival in numerous cancers including melanoma, malignant mesothelioma, prostate cancer, and non-small cell lung cancer cells via c-myc suppression or poly ADP-ribose polymerase (PARP) and Mcl-1 expression increase ${ }^{8-13}$. Importantly, low expression of cytoplasmic PLZF strongly correlated with high tumor grade, lymph node metastasis and indicated a short overall survival (OS) time in nonsmall cell lung cancer ${ }^{14}$. Meanwhile, Hur et al. claimed a tumor-promoting effect of PLZF by repressing the p53 pathway $^{15}$. In thyroid carcinoma, high cytoplasmic 
expression of PLZF was found to be involved in capsular invasion and lymph node metastasis ${ }^{16}$. However, the role of PLZF in GBC has remained to be elucidated.

Interferon-induced protein with tetratricopeptide repeat 2 (IFIT2) is a member of IFN-stimulated genes (ISGs), which are induced after the treatment of type I or III IFNs $^{17}$. It could constitute complexes with itself or with two other related human ISGs, IFIT1 and IFIT3. In addition, IFIT2 has been considered as a tumor suppressor in many tumors to promote cellular apoptosis, suppress tumor proliferation, and metastasis ${ }^{18}$.

In the present study, we provided evidences in vitro and in vivo that PLZF served as a potent tumor suppressor for decreased tumor growth and liver metastasis of GBC. Moreover, IFIT2 expression has been markedly increased following PLZF overexpression, and was demonstrated to be required for the tumor inhibition of PLZF in GBC cells. Therefore, our study demonstrated PLZF reduced GBC progression by IFIT2-dependent p21 increase and suppression of tumor epithelial-mesenchymal transition (EMT). Also, we found PLZF promoted the transcription of IFIT2 by increasing STAT1 protein level. Hence, our study provided a valuable biomarker for prognosis and a potential therapeutic target for GBC.

\section{Materials and methods Tissue samples}

Formalin-fixed, paraffin-embedded (FFPE) tumor samples with histologically confirmed GBC were obtained from 80 patients who had GBC surgical resection and postoperative adjuvant chemotherapy at the Department of Pathology (Renji Hospital) from January 2004 to February 2015. Twenty FFPE gallbladder samples were obtained from gallbladder stone patients. Matched fresh primary GBC samples and relevant non-tumorous tissues were obtained from 15 patients among the $80 \mathrm{GBC}$ patients. All the fresh specimens were routinely snap-frozen in liquid nitrogen. The paraffin-embedded samples for immunohistochemistry (IHC) were evaluated by two certified pathologists at the Department of Pathology. Follow-up data and medical records were collected from the hospital electronic medical records. And the acquisition of patient specimens and medical data got the permission from Ethical Committee of Renji Hospital, Shang Hai Jiao Tong University School of Medicine and the patients or their relatives.

\section{Cell lines}

The human embryonic kidney 293T cells and human GBC cell line GBC-SD were purchased from the Chinese Academy of Life Sciences (Shanghai, China). Another GBC cell line NOZ was kindly gifted by Xinhua hospital (Shanghai, China).

GBC-SD and 293T cells were maintained in RPMI-1640 medium (GibcoBRL, Gaitherburg, MD, USA). NOZ cells were maintained in Willian's E medium. All the cells were maintained in the medium supplemented with $1 \%$ antibiotics and $10 \%$ fetal bovine serum (GIBCO) at $37^{\circ} \mathrm{C}$ with $5 \% \mathrm{CO}_{2}$.

\section{Plasmids construction, transfection, and lentiviral transduction}

Human PLZF expression plasmid was constructed by insertion of the coding sequence (CDS) of PLZF into pCDH-CMV plasmid (System Biosciences, CA, USA). STAT1 plasmid was kindly gifted by Hao Jia from Department of Biochemistry and Molecular Cell Biology, Shanghai Jiaotong University School of Medicine. The PLZF short hairpin RNA (shRNA) was constructed by Shanghai GenePharma Medical Biotechnology Company. The IFIT2 shRNA was constructed by Shanghai GenePharma Medical Biotechnology Company. PCDH-flagPLZF sense: $5^{\prime}$-TGCTCTAGAGCAATGGATTACAAGG ATGACGACGATAAGGATCTGACAAAAATGGGCAT GATC-3'; PCDH-flag-PLZF antisense: 5'-CCGGAATTC CGGTCACACATAGCACAGGTAGAGGTACG-3'. The sense sequence of IFIT2 shRNA was: 5'-CCAAATCCT TCATGTAATA-3'. The sequence of the IFIT2 shRNA was the following: 5'-TGCTGTTGACAGTGAGCGCG CCAAATCCTTCATGTAATATTAGTGAAGCCACAGA TGTAATATTACATGAAGGATTTGGCTTGCCTACTG CCTCGGA-3'. Recombinant lentiviruses were produced using HEK-293T cells and the viruses were harvested and used to infect human GBC cells with $4 \mu \mathrm{g} / \mathrm{ml}$ polybrene (Sigma). Then, the stable expression cells were selected by $2.5 \mu \mathrm{g} / \mathrm{ml}$ puromycin. Mock were these empty vector stably transfected cells and NC were these negative control plasmid stably transfected cells.

\section{RNA extraction and miRNA analysis}

Total RNA was isolated from cell lines using Trizol reagent (Invitrogen) according to the manufacturer's instructions. Complementary DNAs were synthesized using Reverse Transcriptase kit. The gene expression levels were detected by using SYBR Premix Ex Taq (Takara, Shiga, Japan) and performed real-time PCR on the ABI Prism 7500 system (Applied Biosystems, Foster City, CA). Data were normalized to internal control $\beta$-actin.

The primers were PLZF-forward: 5'-TGCGGCTGAG AATGCATTA-3'; PLZF-reverse: 5'-ACACAGCAGACA GAAGACGG-3'. IFIT2-forward: 5'-CTCAAAGGGC AAAACGAGGC-3'; IFIT2-reverse: 5'-CCAGGCATAGT TTCCCCAGG-3'. $\beta$-Actin-forward: 5 '-GGACTTCGAG CAAGAGATGG-3'; $\beta$-actin-reverse: $5^{\prime}$-AGCACTGTGT TGGCGTACAG- 3 '. All the primers above were provided by Biosune Biotech. The experiments were done in triplicates. 


\section{Co-immunoprecipitation (co-IP), protein extraction, and western blot (WB) analysis}

Plasmids encoding STAT1 and PLZF-Flag proteins were transiently transfected into HEK-293T cells, and $48 \mathrm{~h}$ after transfection, cells were lysed in IP lysis buffer with protease inhibitor cocktail and phenylmethanesulfonyl fluoride (PMSF). Co-IP assays were performed with Flag beeds (Sigma-Aldrich; M8823). For normal western blot, total protein was extracted from GBC cells using RIPA lysis buffer supplemented with $1 \%$ PMSF and proteinase inhibitor cocktail. Bicinchoninic acid (BCA) assay was used to measure the protein concentration. Equal amounts of protein were loaded on a $8 \%$ sodium salt -polyacrylamide gel electrophoresis (SDS-PAGE) and transferred to NC membranes (Millipore, Bedford, MA). Then, the blots were blocked in 5\% skimmed milk with $0.1 \%$ Tween 20 for $1 \mathrm{~h}$ at room temperature followed by incubating at $4^{\circ} \mathrm{C}$ overnight with primary antibodies. The membranes were washed with tris-buffered saline and tween (TBST) and then incubated with secondary antibody at room temperature for $2 \mathrm{~h}$. The blots were detected by ECL chemiluminescence kit (Millipore). The PLZF antibody was obtained from Santa Cruz Biotechnology (Santa Cruz, CA). $\beta$-Actin antibody from Abclonal Biotech was used as loading control. Other antibodies were purchased from Proteintech group (Proteintech, USA).

\section{Luciferase gene report assay}

The plasmid containing the IFIT2 promoter region was kindly gifted by Hao Jia from Department of Biochemistry and Molecular Cell Biology, Shanghai Jiaotong University School of Medicine. The IFIT2 promoter and PLZF plasmid were co-transfected into NOZ cells. After 24-h transfection, cells were harvested and the luciferase activities were measured by the Dual-Luciferase Reporter Assay System (Promega). The values of luciferase assay were expressed after normalization to Renilla luciferase activity. Each transfection was carried out in triplicates.

\section{Immunofluorescence}

We also performed immunofluorescence (IF) staining to detect the function of PLZF on IFIT2. In all, $2 \times 10^{4}$ PLZF overexpression cells were seeded to 24-well plate covered with sterile coverslips. The cells were incubated for another $12 \mathrm{~h}$. Then, the primary antibody and matched secondary antibody were adopted to stain PLZF and IFIT2. In all, 4, 6-diamidino-2-phenylindole (DAPI) was used to stain cell nuclei. All assays were performed in triplicates.

\section{CCK-8 and plate colony formation assays}

Cell Counting Kit-8 (CCK-8) and plate colony formation assays were performed to detect the cell proliferation capacity. For CCK-8 assay, 2000 cells with $100 \mu \mathrm{l}$ medium were seeded into each well of 96 -well plates. Considering of the evaporation, periphery wells of 96-well plates did not seed cells. After every $24 \mathrm{~h}, 10 \mu \mathrm{l} \mathrm{CCK-8}$ was added to the medium for $1 \mathrm{~h}$ at $37^{\circ} \mathrm{C}$ and then we measured the absorbance of the plates at $450 \mathrm{~nm}$. For the plate colony formation assay, 600 cells with $2.5 \mathrm{ml}$ medium were seeded into each well of six-well plates and then maintained at $37^{\circ} \mathrm{C}$ with $5 \% \mathrm{CO}_{2}$. After 2-week growth, the cells were fixed and stained with Coomassie Brilliant Blue for 30 min. The number of clones was counted and all the assays were repeated three times.

\section{Cell migration and invasion assays}

Transwell assay was used to assess the migration and invasion abilities of the GBC cells. Twenty-four-well transwell chambers with $8 \mu \mathrm{m}$ pore size polycarbonate membrane (Corning, NY, USA) were used in this assay. For migration assay, $4 \times 10^{4}$ cells with $100 \mu \mathrm{l}$ serum-free medium were seeded into the upper chamber. In the lower chamber, $700 \mu \mathrm{l}$ medium with $10 \%$ fetal bovine serum was added. After $16 \mathrm{~h}$, the migrated cells were fixed with $4 \%$ paraformaldehyde and stained with Coomassie Brilliant Blue. The cells were stained with Coomassie Brilliant Blue. Then, the cells were counted in six random fields of each chamber. For invasion assay, $8 \times 10^{4}$ cells were seeded into the upper chamber with Matrigel (BD)coated membrane for $48 \mathrm{~h}$. All experiments were carried out in triplicates.

\section{Immunohistochemistry}

Specimens fixed in $10 \%$ buffered formalin were made into paraffin-embedded sections. Sections were deparaffinized and rehydrated by dimethylbenzene and ethanol. The antigen activity of specimens was retrieved by $0.01 \mathrm{M}$ sodium citrate buffer ( $\mathrm{pH}$ 6.0). Later, the sections were kept in 3\% hydrogen peroxide for 20 min to erase endogenous peroxidase. Then, the sides were blocked with goat serum and incubated with primary and secondary antibody. 3, 3'-diaminobenzidine (DAB) system was applied in the positive staining. The final results were assessed independently by two pathologists who knew nothing about the group design. The scoring of the staining was based on the intensity and proportion of the positive staining. The staining intensity was classified into four groups $(0=$ negative, $1=$ weak, $2=$ moderate, $3=$ strong); and for the staining area $(0=$ negative, $1=1-9 \%, 2=10-39 \%, 3=40-69 \%$, and $4=70-100 \%)$.

\section{In vivo studies}

To investigate the proliferation effect of PLZF in vivo, $100 \mu \mathrm{l}$ phosphate-buffered saline (PBS) containing $1 \times 10^{6}$ NOZ cells were injected subcutaneously into the right 
lower regions of 4-week-old male nude mice. Tumor length (L) and width (W) were measured every 3 days with vernier caliper. The tumor volume $(\mathrm{V})$ was calculated by the following formula: $\mathrm{V}=\mathrm{L} \times \mathrm{W}^{2} \times 0.5^{19}$. After 5 weeks, all the mice were sacrificed and the subcutaneous xenografts were excised. To further verify the metastatic capability of PLZF in vivo, $100 \mu \mathrm{l}$ PBS containing $5 \times 10^{6}$ $\mathrm{NOZ}$ cells were injected subcutaneously into the nude mice. After 7 weeks from the injection, the mice were sacrificed and the livers were excised and made into sections for hematoxylin and eosin (H\&E) staining and IHC staining. All the animal experiments were approved by the Institutional Review Board of the Renji Hospital of Shanghai Jiao Tong University.

\section{Statistical analysis}

All data were presented as the mean \pm s.d. The variance of each experimental group was determined by an unpaired two-tailed Student's $t$-test. Clinicopathological data were analyzed using Pearson's $X^{2}$ test. Kaplan-Meier method and log-rank test were performed to analyze the survival probabilities. Univariate and multivariate analysis were analyzed using Cox proportional hazard regression model. All of the statistical analyses were performed with SPSS 19.0 software. $P$-values $<0.05$ were considered statistically significant.

\section{Results}

PLZF expression was significantly decreased in GBC and low PLZF expression predicted poor clinical outcome

In 15 pairs of $\mathrm{GBC}$ tissue samples, the mRNA expression levels of PLZF were detected to be lower in GBC tissues compared with that in adjacent non-neoplastic tissues (Fig. 1a). Then, we examined PLZF protein level by performing IHC staining assay in 20 normal gallbladder (NGB) tissues and a tissue microarray including 80 GBC samples. As shown in Fig. 1b, the positive staining of PLZF was mainly observed in the cytoplasm. Compared with NGB tissues, PLZF was significantly decreased in GBC (Fig. 1b). Among the 80 cases of GBC specimens, $61 \%(49 / 80)$ of cases showed lower expression and 39\% (31/80) showed higher expression; whereas in the NGB tissues, 5 (25\%) exhibited lower and 15 (75\%) exhibited higher expression (Fig. 1c). To further investigate the clinical significance of PLZF expression in GBC, we evaluated the correlation between PLZF expression and the clinicopathological characteristics through statistical analysis. Patients were classified into PLZF low and PLZF high groups based on the staining intensity of median expression level of PLZF (PLZF low, score 0,1; PLZF high, score 2,3, Fig. 1d, described in Materials and methods section). The clinicopathological characteristics of the 80 patients were described in Table 1. We found that lower expression of PLZF was correlated with advanced Tumor
Node Metastasis $(\mathrm{TNM})$ stage $(P=0.012)$ and distant metastasis $(P=0.008)$.

Kaplan-Meier survival analysis combined with the logrank test for OS was performed to evaluate the correlation between low PLZF expression and prognosis (Fig. 1e, Table 2), and indicated low expression level of PLZF patients was associated with shorter OS than those with higher PLZF expression (hazard ratio (HR), 0.47; 95\% confidence interval (CI), 0.28-0.80, $P=0.005)$. Moreover, tumor size (HR, 1.71; 95\% CI, 1.05-2.82, $P=0.033)$, TNM stage (HR, 2.27; 95\% CI, 1.36-3.77, $P=0.002)$, lymph node metastasis (HR, 2.16; 95\% CI,1.25-3.72, $P=0.006$ ), and distant metastasis (HR, 2.36; 95\% CI, 1.42-3.91, $P=$ 0.001) were also risk factors for OS. Besides, multivariate analysis using Cox model, including tumor size, TNM stage, lymph node metastasis, distant metastasis, and PLZF expression showed that only PLZF was an independent prognostic factor for OS in GBC patients (HR, 0.48 ; 95\% CI, 0.28-0.83, $P=0.008$, Table 2). The results suggested patients conducting PLZF low expression tended to have a poor prognosis.

\section{PLZF overexpression reduced GBC cells proliferation}

To identify the effect of PLZF on the proliferation of GBC cells, we performed CCK- 8 and colony formation assay in NOZ and GBC-SD cells stably overexpressing PLZF. As shown in Fig. 2a, PLZF-overexpressing cells displayed slower proliferation activity. Furthermore, PLZF reduced the colony formation rates, indicating attenuated colony formation ability. Smaller colonies were also observed in PLZF overexpression cells compared with the MOCK cells, which stably transfected with empty vector (Fig. 2b). Meanwhile, knockdown PLZF expression could significantly enhance the proliferation of GBC cells (Figs. 2c, d) compared with NC group, which stably transfected with negative control plasmid. We also detected whether PLZF could affect the apoptosis of GBC cells, but the apoptosis analysis by flow cytometry results showed no big difference between MOCK and PLZF overexpression cells (Supplement 1). As PLZF has been shown to regulate the downstream genes mainly via binding to the genes' promoter ${ }^{7}$, we performed quantitative real-time PCR (qRT-PCR) to detect the expression changes of genes correlating with cell proliferation and apoptosis, and found p21 was significantly upregulated both in overexpressing PLZF NOZ and GBC-SD cells (Fig. 2e). p21 is mainly regulated by $\mathrm{p} 53$, then we detected the expression of p53 both in mRNA and protein level. The results showed PLZF effect on p21 was independent of p53 (Fig. 2f). As p21 is an important tumor-suppressor gene, PLZF may suppress the proliferation of GBC cells by increasing p21 expression. Collectively, these observations indicated PLZF inhibited GBC cell growth in vitro. 


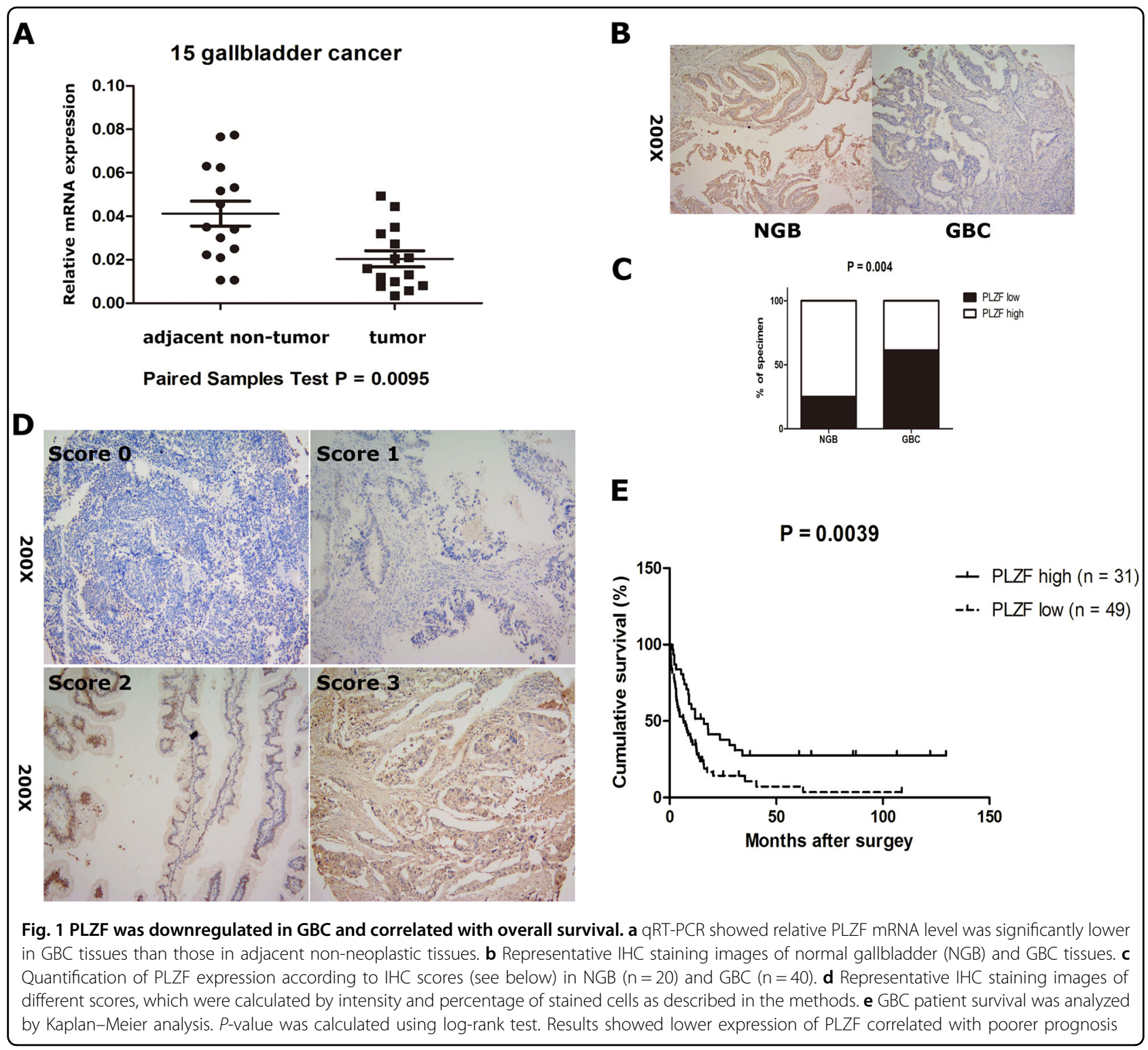

\section{PLZF inhibited migration and invasion of GBC cells}

We next evaluated the migration and invasion ability by transwell migration and matrigel invasion assay. The results showed that the cells on the underside of the filters were significantly decreased in GBC cells overexpressing PLZF compared with mock/vector (Fig. 3a). In addition, we found that PLZF knockdown dramatically enhanced the migration and invasion of GBC cells (Fig. 3b). Moreover, results showed GBC-SD cells underwent morphological change from a rounded or cobblestonelike shape to a spindle shape upon PLZF overexpression (Fig. 3c). As EMT plays crucial role in cancer migration and invasion process, we then detected the mRNA and protein levels of epithelial marker E-cadherin and that of the mesenchymal markers fibronectin, vimentin, and $\mathrm{N}$ - cadherin. The qRT-PCR results revealed that E-cadherin gene expression was prominently increased in PLZF overexpression cells, whereas $\mathrm{N}$-cadherin was reduced (Fig. 3d). Inversely, PLZF knockdown could significantly reduce $\mathrm{E}$-cadherin but increase $\mathrm{N}$-cadherin expression (Fig. 3e). The protein levels of E-cadherin and N-cadherin were shown the same variation features (Figs. 3f, g). Furthermore, we investigated the master transcription factors of EMT, such as Snail, Slug, Twist, Zeb1, and Zeb2 by qRT-PCR. The results showed ectopic PLZF expression could suppress Snail and Slug transcription, and PLZF knockdown showed the opposite effect (Figs. 3d, e). The data presented above suggested PLZF could suppress GBC migratory and invasive abilities by preventing EMT program. 
Table 1 Correlation of PLZF expression with the clinicopathological features of GBC

\begin{tabular}{|c|c|c|c|c|c|}
\hline & \multicolumn{2}{|c|}{ PLZF (high) } & \multicolumn{2}{|c|}{ PLZF (low) } & \multirow[t]{2}{*}{$P$-value } \\
\hline & $N=31$ & $\%$ & $N=49$ & $\%$ & \\
\hline Sexual & & & & & 0.249 \\
\hline Male & 7 & 8.8 & 17 & 21.2 & \\
\hline Female & 24 & 30.0 & 32 & 40.0 & \\
\hline Age (years) & & & & & 0.702 \\
\hline$<65$ & 14 & 17.5 & 20 & 25.0 & \\
\hline$\geq 65$ & 17 & 21.3 & 29 & 36.2 & \\
\hline Tumor size $(\mathrm{cm})$ & & & & & 0.444 \\
\hline$<4$ & 16 & 20.0 & 21 & 26.2 & \\
\hline$\geq 4$ & 15 & 18.8 & 28 & 35.0 & \\
\hline Differential grade & & & & & 0.851 \\
\hline $\mid-\|$ & 19 & 23.8 & 29 & 36.2 & \\
\hline III-IV & 12 & 15.0 & 20 & 25.0 & \\
\hline TNM stage & & & & & $0.012^{*}$ \\
\hline$|-| \mid$ & 19 & 23.8 & 16 & 20.0 & \\
\hline III-IV & 12 & 15.0 & 33 & 41.2 & \\
\hline Lymph node metastasis & & & & & 0.102 \\
\hline No & 26 & 32.5 & 33 & 41.2 & \\
\hline Yes & 5 & 6.3 & 16 & 20.0 & \\
\hline Distant metastasis & & & & & $0.008^{*}$ \\
\hline No & 25 & 31.3 & 25 & 31.2 & \\
\hline Yes & 6 & 7.5 & 24 & 30.0 & \\
\hline
\end{tabular}

\section{PLZF increased IFIT2 expression by activating STAT1 pathway}

To explore the underlying mechanism how PLZF suppressed tumor progression, we analyzed a chip-chip data, which compared PLZF overexpression U937T cells with control U937T cells ${ }^{20}$. Interestingly, a subset of ISGs was significantly increased following PLZF overexpression (Fig. 4a). To confirm the regulation of ISGs expression by PLZF, we checked ISGs expression in PLZF overexpression cells by qRT-PCR. As expected, the transcripts of IFIT2, IFIT1, IFIT3, and TAP1 expression levels were increased both in NOZ and GBC-SD cells (Fig. 4b). Among these ISGs, IFIT2 is a well-established tumor suppressor, which was reported to enhance apoptosis, inhibit proliferation, migration, and invasion in numerous cancers. The expression correlation between PLZF and IFIT2 suggested that PLZF effect on GBC proliferation, migration, and invasion might be mediated by regulating IFIT2. We started by verifying IFIT2 protein levels in PLZF overexpression cells. WB and IF staining showed PLZF increased IFIT2 protein levels both in NOZ and GBC cells (Figs. 4c, d). Next, we performed luciferase report assay to evaluate if PLZF might regulate IFIT2 at transcriptional level. The pGL3 vector encoding the region of IFIT2 promoter and different amount of PLZF plasmids were co-transfected into NOZ cells. We found the reporter activity of IFIT2 was proportionally increased in correspondence with the amount of PLZF (Fig. 4e), suggesting PLZF might serve as a transcriptional activator of IFIT2. Moreover, we evaluated the clinical relevance of PLZF and IFIT2 in GBC specimens by IHC staining assay. Like PLZF, IFIT2 exhibited relatively low expression in GBC specimens (Fig. 4f). Seven of $10 \mathrm{GBC}$ tissues were demonstrated weak or negative staining of IFIT2, whereas

Table 2 Univariate and multivariate analysis of the correlation of prognosis with PLZF and clinicopathologic data in GBC

\begin{tabular}{|c|c|c|c|c|}
\hline \multirow[t]{2}{*}{ Variable } & \multicolumn{2}{|c|}{ Univariable analysis } & \multicolumn{2}{|c|}{ Multivariable analysis } \\
\hline & HR $(95 \% \mathrm{Cl})$ & $P$-value & HR $(95 \% \mathrm{Cl})$ & $P$-value \\
\hline Sex (male vs. female) & $1.13(0.67-1.92)$ & 0.646 & & \\
\hline Age (<65 vs. $\geq 65)$ & $1.24(0.76-2.02)$ & 0.39 & & \\
\hline Histological grade (III-IV vs. I-II) & $1.18(0.72-1.94)$ & 0.501 & & \\
\hline Tumor size ( $\geq 4$ vs. $<4$ ) & $1.71(1.05-2.82)$ & $0.033^{*}$ & $1.32(0.75-2.33)$ & 0.328 \\
\hline TNM stage (III-IV vs. I-II) & $2.27(1.36-3.77)$ & $0.002^{*}$ & $1.47(0.73-2.94)$ & 0.281 \\
\hline Lymph node metastasis (presence vs. absence) & $2.16(1.25-3.72)$ & $0.006^{*}$ & $1.41(0.68-2.94)$ & 0.359 \\
\hline Distant metastasis (presence vs. absence) & $2.36(1.42-3.91)$ & $0.001^{*}$ & $1.20(0.55-2.63)$ & 0.648 \\
\hline PLZF expression (high vs. low) & $0.47(0.28-0.80)$ & $0.005^{*}$ & $0.48(0.28-0.83)$ & $0.008^{*}$ \\
\hline
\end{tabular}

$H R$ hazard ratio; $\mathrm{Cl}$ confidence interval

$P<0.05$ was considered statistically significant, ${ }^{*} P<0.05$. 


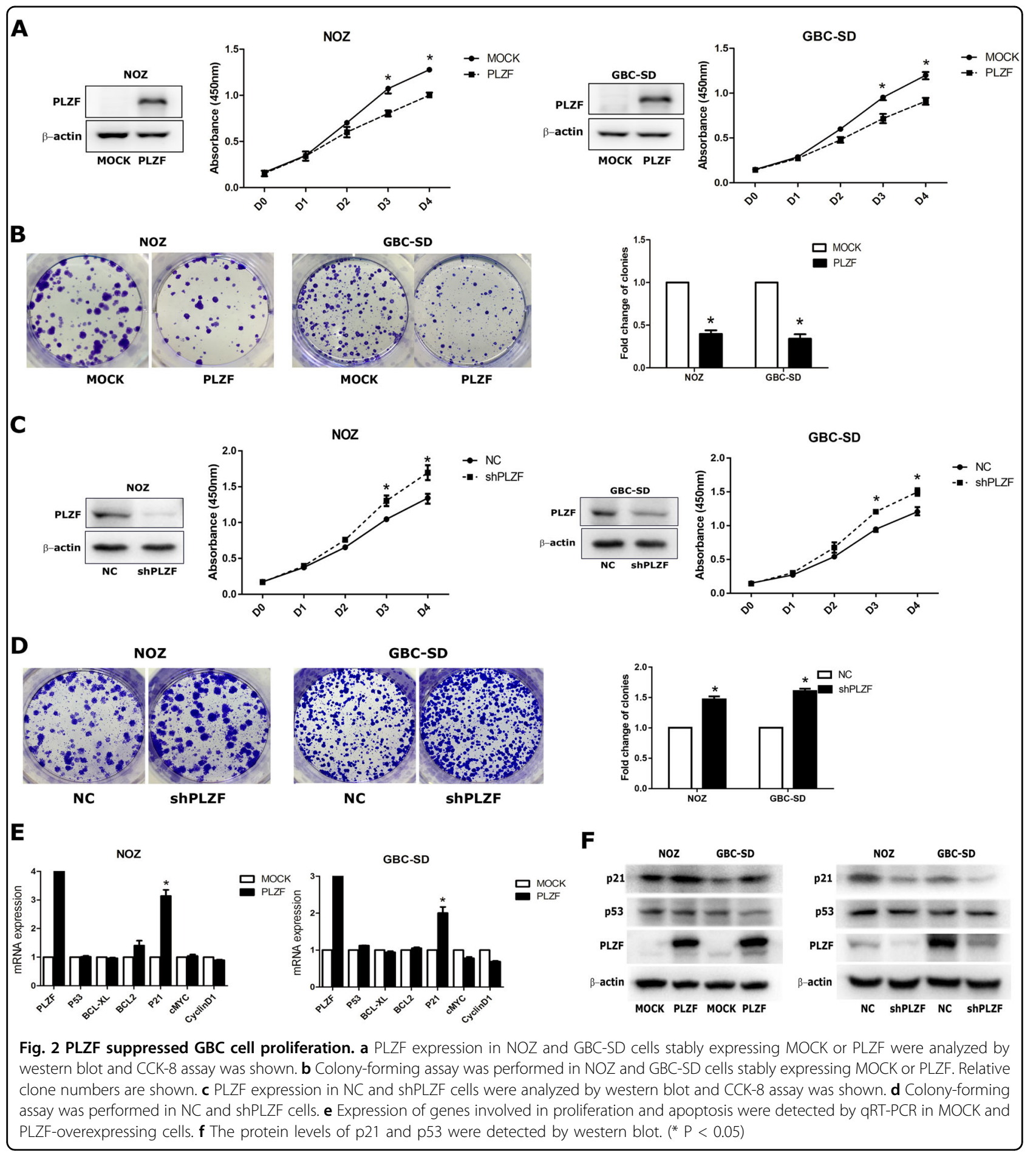

$30 \%$ cases showed IFIT2 high expression. In addition, PLZF and IFIT2 mRNA expression in 15 GBC tissues were detected, which showed PLZF was positively correlated with IFIT2 (Fig. 4g).

To elucidate the molecular mechanism by which PLZF affects IFIT2 expression, we detected the expression of
STAT1 and STAT3 by western blotting. Strikingly, total STAT1 and phosphorylation of STAT1 was significantly enhanced in PLZF overexpression cells compared with control cells after the stimulation of IFN $\gamma$, but there was no change of total STAT3 and phosphorylation of STAT3 (Fig. 4h). Also, we detected the mRNA levels of STAT1 $\alpha$ 


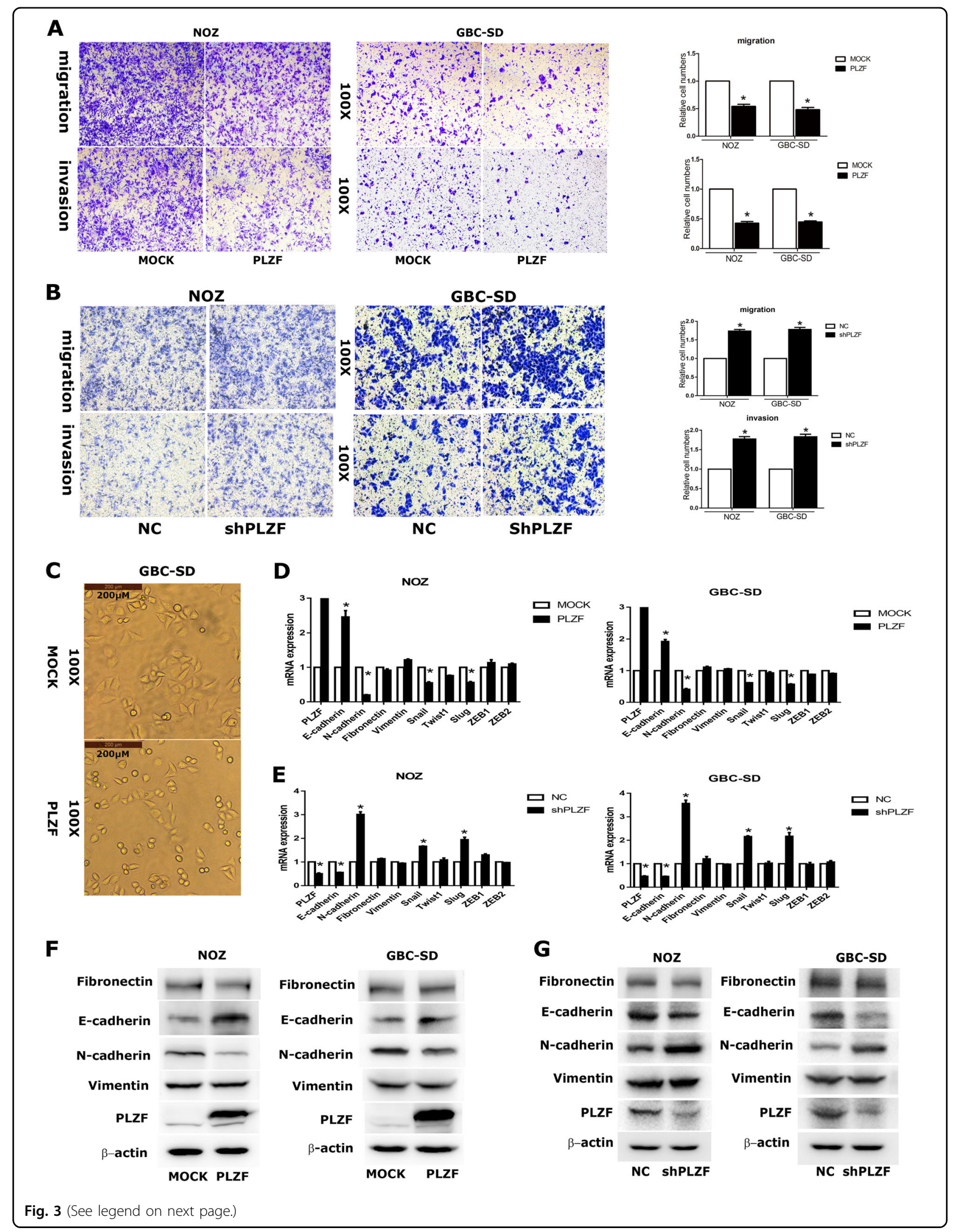


and STAT1 $\beta$ following PLZF overexpression or PLZF downregulation by qRT-PCR and the results showed no difference (Supplement 2). Then, we performed co-IP assay in HEK-293T cells, which transfected with STAT1 and PLZF-Flag plasmids and results showed STAT1 could interacted with PLZF protein (Fig. 4i). These results, taken together, indicated that PLZF promoted the transcription of IFIT2 by increasing STAT1 protein level.

\section{PLZF suppressed the proliferation and invasion of GBC cells via IFIT2}

As we have verified PLZF could regulate the expression of IFIT2, we assumed that the function of PLZF might be dependent on IFIT2. Hence, we depleted IFIT2 expression by siRNA-IFIT2 (siIFIT2) transfection in control group and PLZF-overexpressing NOZ cells, followed by examining GBC cell proliferation and migration. Figures $5 \mathrm{a}$, b showed IFIT2 knockdown significantly promoted cell growth and rescued the tumor inhibitory effects following PLZF overexpression. Additionally, evaluation of GBC cell migration and invasiveness capability showed IFIT2 silencing remarkably triggered migration and invasion, whereas the function of PLZF on migration and invasion was abolished by IFIT2 ablation (Fig. 5c). The similar results were obtained in GBC-SD cells (Figs. 4d-f). In accord with the altered phenotypes, IFIT2 depletion rescued the decreased expression of N-cadherin, but suppressed E-cadherin and p21 expression (Figs. 5g-j). Taken together, the results suggested PLZF inhibited proliferation, migration, and invasion through IFIT2 pathway.

\section{PLZF was essential for primary tumor formation and metastasis of GBC}

Given that we demonstrated the tumor repressive role of PLZF in vitro, we next determined whether PLZF suppressed GBC cell growth and metastasis in vivo. GBC cells with stable expression of PLZF or control vector were subcutaneously implanted into nude mice. The tumor volumes were measured every 3 days. After 5 weeks, the mice were sacrificed and xenograft tumors were removed and analyzed. As shown in Fig. 6a, the tumors originated from PLZF overexpression cells were remarkably smaller than the control group, whereas PLZF overexpression group had smaller tumor volume and tumor weight (Figs. 6b-d). For metastasis assay, after approximately 7 weeks post injection, the mice organs were harvested for $H \& E$ analysis. Multiple lesions in livers were observed in control mice. Surprisingly, a few nodes were observed in mice livers ectopically expressing PLZF (Fig. 6e). H\&E staining confirmed less metastatic nodes in mice livers of PLZF overexpression compared with the control group (Fig. 6f). Importantly, we also found PLZFoverexpressing group had low expression of $\mathrm{N}$-cadherin and high expression of E-cadherin compared with control group (Fig. 6g). The IHC staining of liver metastatic nodes from nude mice further proved PLZF regulated EMT. In summary, PLZF suppressed tumor growth and metastasis in nude mouse model.

\section{Discussion}

GBC is a malignant cancer with very poor prognosis because of its high potential to metastasize in a short time $^{21}$. But the mechanism of tumor proliferation and metastasis is still poorly understood. Cumulative evidences have shown PLZF was downregulated in numerous cancers and relevant with the clinical prognosis. Moreover, the functions of PLZF in cancers remained to be ambiguous and open to interpretations of either a tumor suppressor or tumor promoter as reported previously ${ }^{7}$. However, little is known about the role of PLZF in GBC progression. In this study, the mRNA transcripts of PLZF were significantly downregulated in GBC tissues in contrast to the adjacent counterparts. Consistently, the protein levels of PLZF were reduced in many GBC tissues. Moreover, the decreased PLZF was demonstrated to be associated with tumor size, TNM stage, lymph node metastasis, and distant metastasis. Multivariate analysis using Cox's regression suggested that PLZF was an independent prognostic factor for OS in GBC patients. In agreement with its role in clinical samples, PLZF was further found to suppress GBC cell growth and metastatic activity in vitro, supporting PLZF being a potential tumor suppressor in GBC.

In this study, we found PLZF overexpression could suppress cell proliferation and tumor growth. Furthermore, we investigated the genes involved in tumor growth and found PLZF could increase p21 mRNA level, but no impact on the apoptotic genes like p53, BCL-2, BCL-XL,

\footnotetext{
(see figure on previous page)

Fig. 3 PLZF suppressed GBC cells migration and invasion through EMT. a Transwell migration and matrigel invasion assays were conducted in cells stably expressing MOCK or PLZF. The relative cell numbers were shown. $\mathbf{b}$ Transwell migration and matrigel invasion assays were conducted in NC and shPLZF cells. Relative cell numbers are shown. c GBC-SD cells underwent morphological change from a rounded or cobblestone-like shape to a spindle shape upon PLZF overexpression. $\mathbf{d}$ Expression of epithelial marker E-cadherin, mesenchymal markers fibronectin, vimentin, and N-cadherin were detected by qRT-PCR in MOCK and PLZF-overexpressing cells. e Expression of EMT markers were detected by qRT-PCR in NC and shPLZF cells. $\mathbf{f}$ Expression of epithelial marker E-cadherin, mesenchymal markers fibronectin, vimentin, and N-cadherin were detected by western blot in MOCK and PLZF-overexpressing cells. $\mathbf{g}$ Expression of EMT markers were detected by western blot in NC and shPLZF cells. $(P<0.05)$
} 


\begin{tabular}{|c|c|c|}
\hline Gene Symbol & p value & Foll Change \\
\hline IFIT2 & 0.00000582 & 17.56893 \\
\hline IFI16 & 0.00000682 & 15.69888 \\
\hline IRF2 & 0.0000536 & 5.722638 \\
\hline ISGF3G & 0.000256 & 3.754407 \\
\hline TRIM23 & 0.0000481 & 3.172582 \\
\hline IRF1 & 0.000334 & 2.955347 \\
\hline IFIT3 & 0.0000549 & 2.724262 \\
\hline TAP1 & 0.0000171 & 2.501976 \\
\hline STAT2 & 0.000131 & 2.441797 \\
\hline STAT6 & 0.002516 & 1.586136 \\
\hline TRIM33 & 0.009774 & 1.541402 \\
\hline
\end{tabular}

C

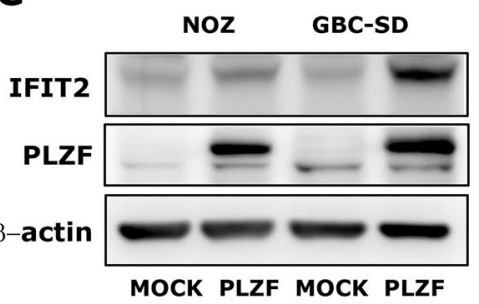

E

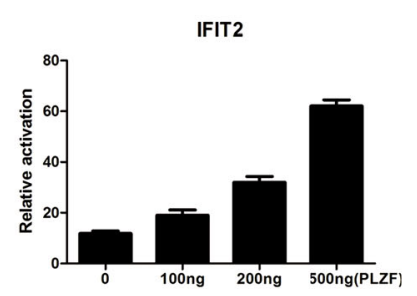

B
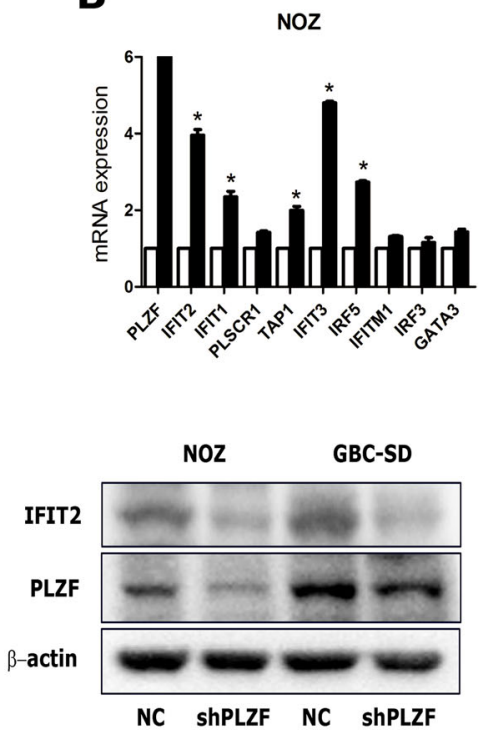

D

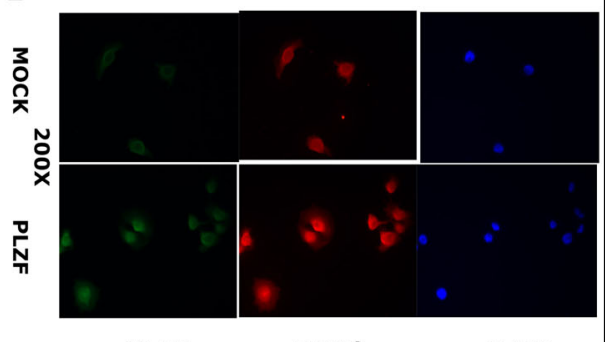

PLZF

IFIT2

DAPI

G
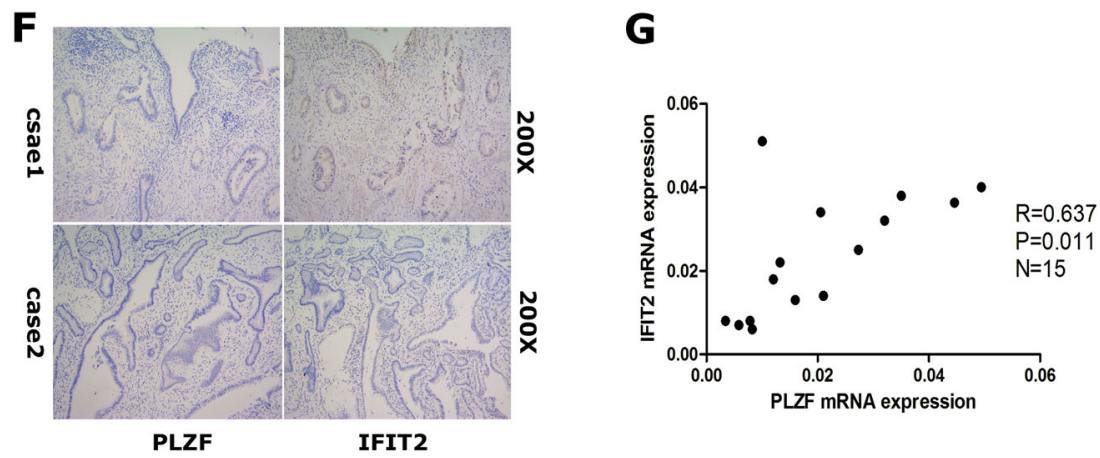

I
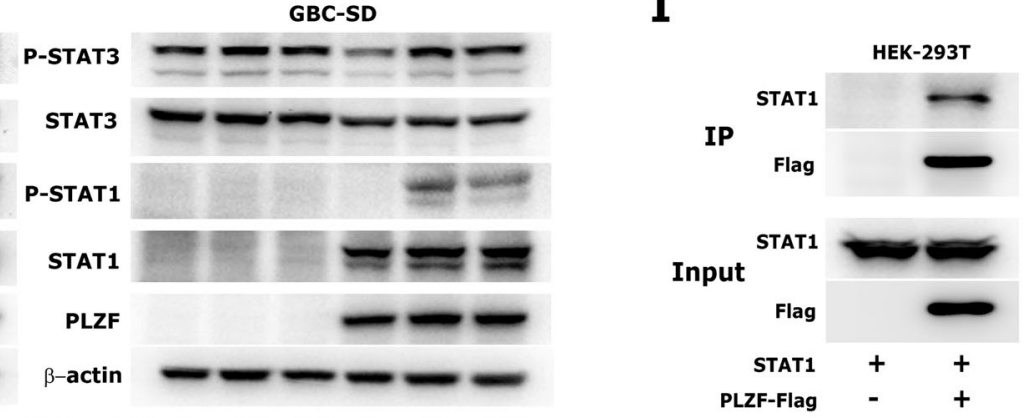

$\beta-$ actin

PLZF-Flag

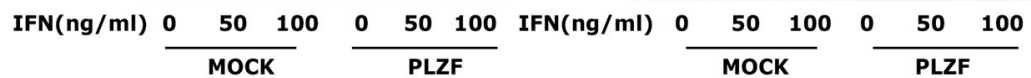

H

NOZ

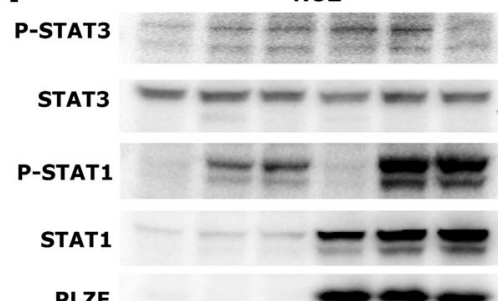

PLZF

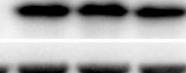

Fig. 4 PLZF increased IFIT2 mRNA and protein expression. a A chip-chip data comparing PLZF-overexpressing cells with control showed PLZF regulated a subset of ISGs. $\mathbf{b}$ Expression of ISGs were detected by qRT-PCR in NOZ and GBC-SD cells stably expressing MOCK or PLZF. c IFIT2 expression was detected by western blot in PLZF-overexpressing and knockdown cells. d IFIT2 expression was detected by IF staining in NOZ cells stably expressing MOCK or PLZF. e IFIT2 promoter reporter and various amount of PLZF plasmids were co-transfected into NOZ cells. The reporter activity of IFIT2 was measured by luciferase assay. $\mathbf{f}$ Representative IHC staining images of PLZF and IFIT2 in two GBC cases. $\mathbf{g}$ Expression of PLZF and IFIT2 in the 15 GBC tissues were positively correlated $(R=0.637, P=0.011)$. $\mathbf{h}$ Ectopic expression of PLZF in GBC cells increased STAT1 and phosphorylation of STAT1 when treated with IFNY. The protein levels of STAT1, pSTAT1, STAT3, and pSTAT3 were detected by western blotting. i Co-IP assay using Flag beeds was performed in HEK-293T cells, which transfected with STAT1 and PLZF-Flag plasmids. (P < 0.05) 
A

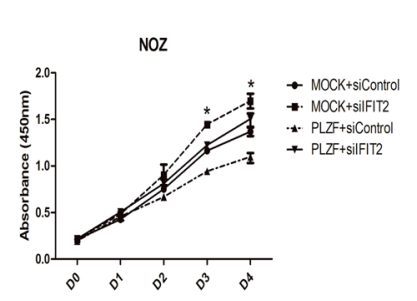

B MOCK+sicontrol MOCK+siIFIT2

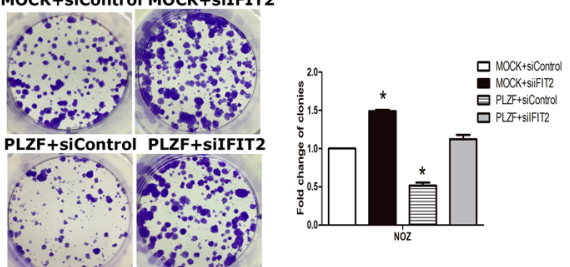

C
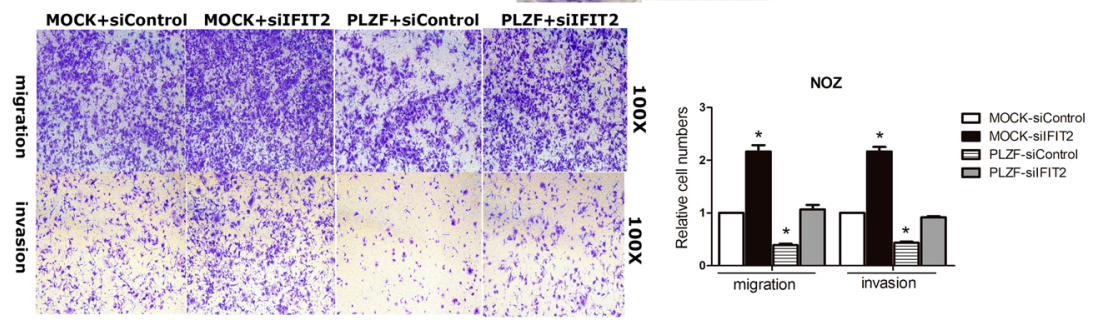

D

E
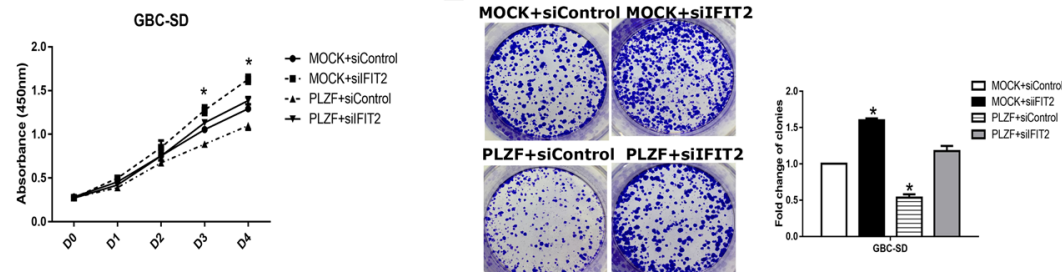

F MOCK+siControl MOCK+siIFIT2 PLZF+sicontrol PLZF+siIFIT2

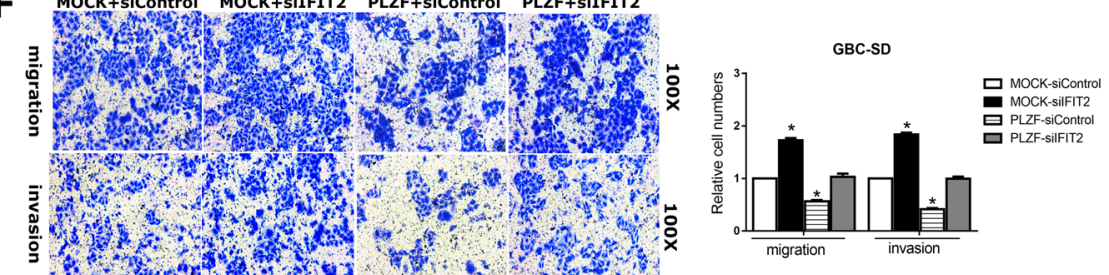

G

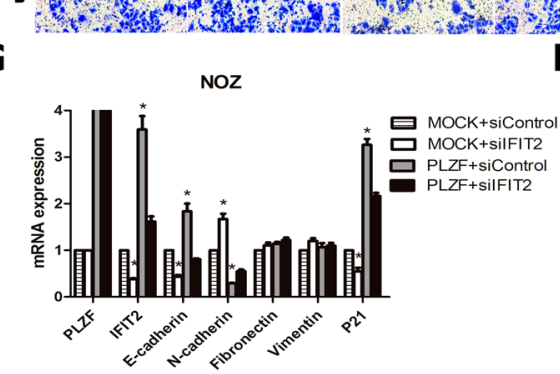

I

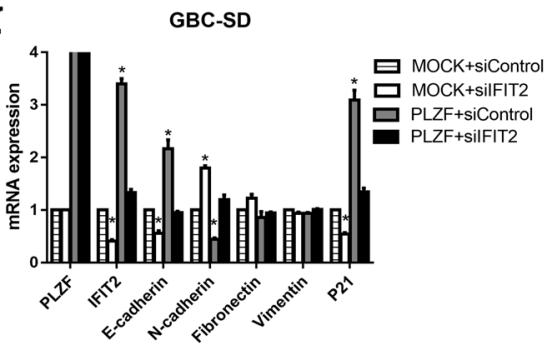

H.

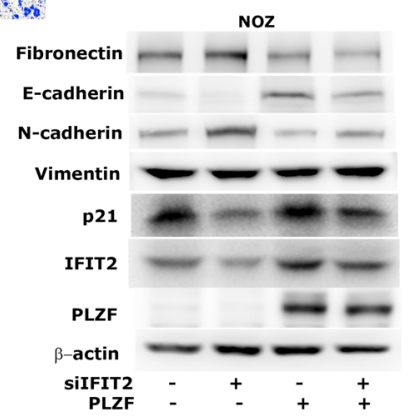

J

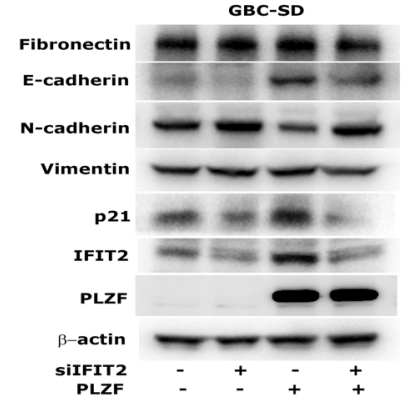

Fig. 5 (See legend on next page.) 
(see figure on previous page)

Fig. 5 IFIT2 knockdown reversed the function of PLZF in NOZ cells. a, d CCK-8 assay was conducted in NOZ and GBC-SD cells transfected with $\mathrm{MOCK}+$ siControl, MOCK + silFIT2, PLZF + siControl and PLZF + silFIT2. b, e Colony formation assay was conducted in NOZ and GBC-SD cells transfected with MOCK + siControl, MOCK + silFIT2, PLZF + siControl and PLZF + silFIT2. Relative clone numbers are shown. c, f Transwell migration and matrigel invasion assays were conducted in NOZ cells transfected with MOCK + siControl, MOCK + silFIT2, PLZF + siControl and PLZF + silFIT2. Relative cell numbers are shown. $\mathbf{g}$, i Expression of EMT markers and p21 were detected by qRT-PCR in GBC cells transfected with MOCK + siControl, MOCK + silFIT2, PLZF + siControl and PLZF + silFIT2. $\mathbf{h}, \mathbf{j}$ Expression of EMT markers and p21 were detected by western blot. $(P<0.05)$

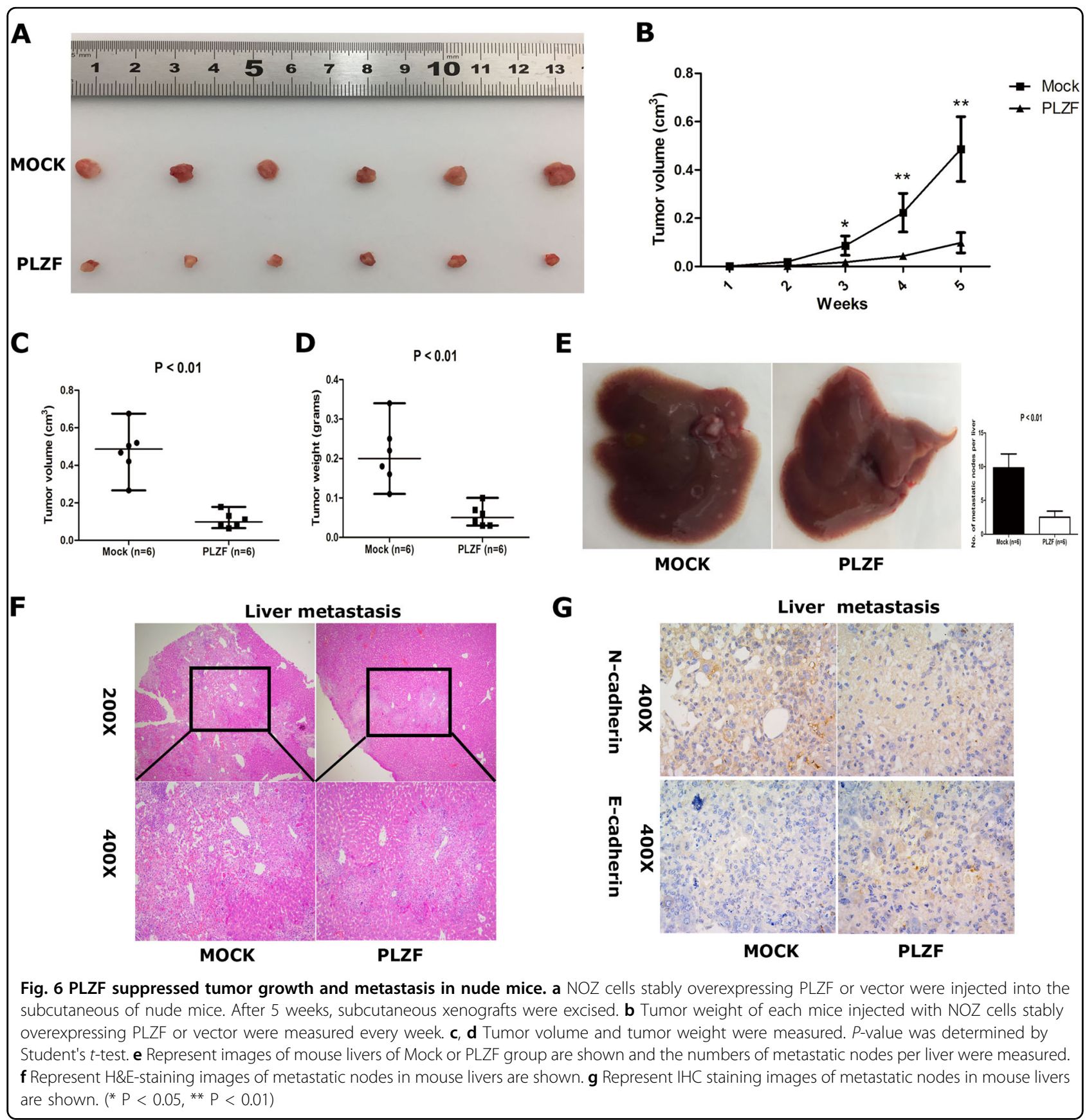


and BAX. p21 is a well-studied gene required for cell cycle arrest. Reduced p21 expression was considered as an early event in gallbladder carcinogenesis ${ }^{22}$. Lots of studies have shown p21 has low expression in GBC tissues and absence of p21 expression independently predicts poor outcome $^{23,24}$. Moreover, silence of $\mathrm{p} 21$ has been proved to promote GBC cells (NOZ and GBC-SD) proliferation significantly ${ }^{25}$. It is involved in cell proliferation inhibition in both p53-dependent and -independent way ${ }^{26}$. A previous study showed PLZF could interact with some corepressors and bind to p21 promoter to repress its transcription ${ }^{15}$. But our results showed opposite phonotypes. This could be due to the fact that the cells we used were different from those studied or the regulation of PLZF might be context dependent.

EMT is regarded as a potent driver conferring cells with metastatic features, including lower expression of epithelial markers like E-cadherin and higher expression of mesenchymal markers like $\mathrm{N}$-cadherin, vimentin, and Fibronectin ${ }^{27-30}$. EMT gets cells to obtain strong capability of motility and spreading, leading to metastasis to the distant regions ${ }^{31,32}$. The underlying molecular mechanism of PLZF in cancer metastasis remained little understood. Only one study in melanoma demonstrated PLZF suppressed migration and invasion partially through integrin $\beta 3$ and MMP9 $^{9}$. We examined the expression levels of EMT markers for possible mechanism investigations, which showed PLZF overexpression decreased Ncadherin expression but enhanced E-cadherin level, whereas vimentin and fibronectin were not altered, suggesting PLZF could selectively induce mesenchymal markers for initiating EMT program.

To clarify the underlying mechanism of PLZF, we performed chip-chip data analysis using online database and found a link between PLZF and ISGs. Through highaffinity binding with cell surface receptors, IFN molecules can activate the Janus kinase/Signal Transducer and Activator of Transcription (JAK/STAT) signaling pathway, resulting in induction of thousands of ISGs at the transcript level ${ }^{33-35}$. Growing studies have verified ISGs are involved in multiple processes including apoptosis, angiogenesis, and metastasi ${ }^{33-37}$. To validate this prediction, qRT-PCR analysis was used and showed that PLZF overexpression enhanced the expression of a subset of ISGs included IFIT2 in particular. This observation was also confirmed by WB and IF analysis, indicating PLZF could regulate IFIT2 mRNA transcription. Dual-luciferase reporter assays were then performed to further verify PLZF effect on IFIT2 expression regulation. The results showed PLZF increased IFIT2 in a dose-dependent manner. IHC staining of PLZF and IFIT2 in GBC tissues also confirmed a strong correlation. Accumulating evidences have established IFIT2 as a tumor suppressor in various tumor types. IFIT2 promotes apoptosis through the intrinsic apoptosis mechanism by regulating antiapoptotic and proapoptotic factors ${ }^{17,38-41}$. Moreover, IFIT2 was reported to suppress the migration and metastasis by regulating atypical protein kinase $\mathrm{C}$, leading to EMT and cell migration ${ }^{42}$. We therefore verified whether IFIT2 was required for the function of PLZF. We introduced IFIT2 small interfering RNA into PLZF overexpression cells and found the expression of p21 and E-cadherin was decreased, but $\mathrm{N}$-cadherin was increased in PLZF overexpression cells with IFIT2 silencing. Moreover, downregulation IFIT2 expression could rescue the inhibitory effects of proliferation, migration, and invasion in PLZF overexpression cells. IFNs exert their biological effects by activating STATs expression, which translocate into the nuclear and bind ISGs to transactivate their activation ${ }^{43}$. STAT1 is the central mediator of ISGs' promotion ${ }^{44}$. Notably, we showed that ectopic PLZF expression could enhance STAT1 and pSTAT1 after IFN $\gamma$ treatment, indicating that PLZF may function as a suppressor of STAT1/ IFIT2 pathway. Moreover, our results showed PLZF could interact with STAT1 protein and increase its protein level by an unknow mechanism. Previous studies have demonstrated that STAT1 is mainly degraded by ubiquitination and proteasome pathway ${ }^{45}$. So PLZF might inhibit STAT1 degradation in that way. Collectively, these results suggested PLZF might function concomitantly with IFIT2 for decreased GBC cell proliferation, migration and invasion.

Above all, we revealed that PLZF was remarkably reduced in GBC tissues and low PLZF expression was correlated with advanced TNM stage, distant metastasis, and short overall survival. Furthermore, we found PLZF overexpression inhibited tumor growth and metastasis by activating STAT1/IFIT2 pathway in GBC cells and nude mice. To our knowledge, we are the first to identify the tumor-suppressor role of PLZF in GBC both in vitro and in vivo. PLZF might be a potential tumor biomarker and a critical therapeutic target for GBC.

\section{Acknowledgements}

This work was supported by National Science Foundation of China (81272748, 81472240, and 81072011), Science and Technology Commission of Shanghai Municipality (16411952700, 10411955400, and 09411960800), Program for Outstanding Academic Leader of Shanghai (2016, JW).

Author contributions

J.W. designed this study. H.S., M.Z., Y.Z., S.H., S.X., and X.H. performed the experiments. M.H. provided materials. H.S., M.Z., Y.Y., and M.M. analyzed the data. H.S., Y.Z., Y.Y., M.M., and J.W. wrote the manuscript.

Conflict of interest

The authors declare that they have no conflict of interest.

\section{Publisher's note}

Springer Nature remains neutral with regard to jurisdictional claims in published maps and institutional affiliations. 


\section{Supplementary information}

The online version of this article (https://doi.org/10.1038/s41419-017-0107-3) contains supplementary material.

Received: 23 May 2017 Revised: 23 October 2017 Accepted: 24 October 2017

Published online: 22 January 2018

\section{References}

1. Jemal, A., Siegel, R., Xu, J. \& Ward, E. Cancer statistics, 2010. CA. Cancer. J. Clin. 60, 277-300 (2010).

2. Kanthan, R., Senger, J. L., Ahmed, S. \& Kanthan, S. C. Gallbladder cancer in the 21st century. J. Oncol. 2015, 967472 (2015).

3. Bartlett, D. L., Fong, Y., Fortner, J. G., Brennan, M. F. \& Blumgart, L. H. Long-term results after resection for gallbladder cancer. Implications for staging and management. Ann. Surg. 224, 639-646 (1996).

4. Wullstein, C., Woeste, G., Barkhausen, S., Gross, E. \& Hopt, U. T. Do complications related to laparoscopic cholecystectomy influence the prognosis of gallbladder cancer? Surg. Endosc. 16, 828-832 (2002).

5. Spicuglia, S. et al. Characterisation of genome-wide PLZF/RARA target genes. PLOS ONE 6, e24176 (2011)

6. Licht, J. D. et al. Clinical and molecular characterization of a rare syndrome of acute promyelocytic leukemia associated with translocation (11:17). Blood $\mathbf{8 5}$ 1083-1094 (1995)

7. Liu, T. M., Lee, E. H., Lim, B. \& Shyh-Chang, N. Concise review: balancing stem cell self-renewal and differentiation with PLZF. Stem Cells (Dayton, Ohio) 34, 277-287 (2016)

8. McConnell, M. J. et al. Growth suppression by acute promyelocytic leukemiaassociated protein PLZF is mediated by repression of c-myc expression. Mol. Cell. Biol. 23, 9375-9388 (2003).

9. Felicetti, F. et al. Role of PLZF in melanoma progression. Oncogene 23, 4567-4576 (2004)

10. Cheung, M. et al. The promyelocytic leukemia zinc-finger gene, PLZF, is frequently downregulated in malignant mesothelioma cells and contributes to cell survival. Oncogene 29, 1633-1640 (2010).

11. Jin, $Y$. et al. Molecular circuit involving KLK4 integrates androgen and mTOR signaling in prostate cancer. Proc. Natl. Acad. Sci. USA 110 E2572-E2581 (2013).

12. Wang, $X$. et al. Hypermethylation reduces expression of tumor-suppresso PLZF and regulates proliferation and apoptosis in non-small-cell lung cancers. FASEB J. 27, 4194-4203 (2013).

13. Cao, J. et al. PLZF mediates the PTEN/AKT/FOXO3a signaling in suppression of prostate tumorigenesis. PLOS ONE 8, e77922 (2013).

14. Xiao, G. Q. et al. Down-regulation of cytoplasmic PLZF correlates with high tumor grade and tumor aggression in non-small cell lung carcinoma. Hum. Pathol. 46, 1607-1615 (2015)

15. Choi, W. I. et al. Role of promyelocytic leukemia zinc finger (PLZF) in cell proliferation and cyclin-dependent kinase inhibitor 1A (p21WAF/CDKN1A) gene repression. J. Biol. Chem. 289, 18625-18640 (2014).

16. Matsuzawa, $\mathrm{K}$ et al. Implication of intracellular localization of transcriptional repressor PLZF in thyroid neoplasms. BMC Endocr. Disord. 14, 52 (2014).

17. Reich, N. C. A death-promoting role for ISG54/IFIT2. J. Interferon Cytokine Res. 33, 199-205 (2013).

18. $\mathrm{Xu}, \mathrm{D}$. et al. Promyelocytic leukemia zinc finger protein regulates interferonmediated innate immunity. Immunity 30, 802-816 (2009).

19. Tomayko, M. M. \& Reynolds, C. P. Determination of subcutaneous tumor size in athymic (nude) mice. Cancer Chemother. Pharmacol. 24, 148-154 (1989).

20. Rice, K. L. et al. Comprehensive genomic screens identify a role for PLZFRARalpha as a positive regulator of cell proliferation via direct regulation of cMYC. Blood 114, 5499-5511 (2009).
21. Hundal, R. \& Shaffer, E. A. Gallbladder cancer: epidemiology and outcome. Clin. Epidemiol. 6, 99-109 (2014).

22. Li, X., Hui, A. M., Shi, Y. Z., Takayama, T. \& Makuuchi, M. Reducedp21(WAF1/ CIP1) expression is an early event in gallbladder carcinogenesis and is of prognostic significance for patients with carcinomas of the gallbladder. Hum. Pathol. 32, 771-777 (2001)

23. Kim, W. B. et al. Expression and clinical significance of cell cycle regulatory proteins in gallbladder and extrahepatic bile duct cancer. Ann. Surg. Oncol. 16, 23-34 (2009).

24. Alsheyab, F. M., Ziadeh, M. T. \& Bani-Hani, K. E. Expression of p21 and p27 in gallbladder cancer. Saudi. Med. J. 28, 683-687 (2007).

25. Cai, Q. et al. Long non-coding RNA UCA1 promotes gallbladder cancer progression by epigenetically repressing p21 and E-cadherin expression. Oncotarget 8, 47957-47968 (2017)

26. Lohr, K., Moritz, C., Contente, A. \& Dobbelstein, M. p21/CDKN1A mediates negative regulation of transcription by p53. J. Biol. Chem. 278, 32507-32516 (2003).

27. De Craene, B. \& Berx, G. Regulatory networks defining EMT during cance initiation and progression. Nat. Rev. Cancer 13, 97-110 (2013).

28. Ombrato, L \& Malanchi, I. The EMT universe: space between cancer cell dissemination and metastasis initiation. Crit. Rev. Oncog. 19, 349-361 (2014).

29. Guan, X. Cancer metastases: challenges and opportunities. Acta Pharmaceutica Sinica B 5, 402-418 (2015).

30. Guarino, M. Epithelial-mesenchymal transition and tumour invasion. Int. J. Biochem. Cell. Biol. 39, 2153-2160 (2007).

31. Thiery, J. P. \& Sleeman, J. P. Complex networks orchestrate epithelialmesenchymal transitions. Nat. Rev. Mol. Cell. Biol. 7, 131-142 (2006).

32. Huber, M. A., Kraut, N. \& Beug, H. Molecular requirements for epithelialmesenchymal transition during tumor progression. Curr. Opin. Cell. Biol. 17 548-558 (2005)

33. de Veer, M. J. et al. Functional classification of interferon-stimulated genes identified using microarrays. J. Leukoc. Biol. 69, 912-920 (2001).

34. Stark, G. R. \& Darnell, J. E. Jr. The JAK-STAT pathway at twenty. Immunity $\mathbf{3 6}$ 503-514 (2012).

35. Gongora, C. \& Mechti, N. [Interferon signaling pathways]. Bull. Cancer 86, 911-919 (1999).

36. Cheon, H., Borden, E. C. \& Stark, G. R. Interferons and their stimulated genes in the tumor microenvironment. Semin. Oncol. 41, 156-173 (2014).

37. Fish, E. N. \& Platanias, L. C. Interferon receptor signaling in malignancy: a network of cellular pathways defining biological outcomes. Mol. Cancer Res. 12, 1691-1703 (2014).

38. Stawowczyk, M., Van Scoy, S., Kumar, K. P. \& Reich, N. C. The interferon stimulated gene 54 promotes apoptosis. J. Biol. Chem. 286, 7257-7266 (2011)

39. Jia, $\mathrm{H}$. et al. The LIM protein AJUBA promotes colorectal cancer cell survival through suppression of JAK1/STAT1/IFIT2 network. Oncogene 36, 2655-2666 (2017).

40. Wang, Y. et al. Long non-coding RNA LINC00161 sensitises osteosarcoma cells to cisplatin-induced apoptosis by regulating the miR-645-IFIT2 axis. Cancer Lett 382, 137-146 (2016)

41. Feng, $X$. et al. MicroRNA-645, up-regulated in human adencarcinoma of gastric esophageal junction, inhibits apoptosis by targeting tumor suppressor IFIT2. BMC. Cancer 14, 633 (2014).

42. Lai, K. C., Liu, C. J., Chang, K. W. \& Lee, T. C. Depleting IFIT2 mediates atypical PKC signaling to enhance the migration and metastatic activity of ora squamous cell carcinoma cells. Oncogene 32, 3686-3697 (2013).

43. Schneider, W. M., Chevillotte, M. D. \& Rice, C. M. Interferon-stimulated genes: a complex web of host defenses. Annu. Rev. Immunol. 32 513-545 (2014).

44. Blouin, C. M. \& Lamaze, C. Interferon gamma receptor: the beginning of the journey. Front. Immunol. 4, 267 (2013).

45. Yuan, C., Qi, J., Zhao, X. \& Gao, C. Smurf1 protein negatively regulates interferon-gamma signaling through promoting STAT1 protein ubiquitination and degradation. J. Biol. Chem. 287, 17006-17015 (2012). 